Pacific Journal of Mathematics

GENERALIZATIONS OF SHANNON-MCMILLAN THEOREM 


\title{
GENERALIZATIONS OF SHANNON-MCMILLAN THEOREM
}

\author{
Shu-Teh C. MoY
}

1. Introduction. Let $X$ be a non-empty set and $\mathscr{S}$ be a $\sigma$-algebra of subsets of $X$. Consider the infinite product space $\Omega=\prod_{n=-\infty}^{\infty} X_{n}$ where $X_{n}=X$ for $n=0, \pm 1, \pm 2, \cdots$ and the infinite product $\sigma$-algebra $\mathscr{F}=\prod_{n=-\infty}^{\infty} \mathscr{S}_{n}$ where $\mathscr{S}_{n}=\mathscr{S}$ for $n=0, \pm 1, \pm 2, \cdots$. Elements of $\Omega$ are bilateral infinite sequences $\left\{\cdots, x_{-1}, x_{0}, x_{1}, \cdots\right\}$ with $x_{n} \in X$. Let us denote the elements of $\Omega$ by $\omega$. If $\omega=\left\{\cdots, x_{-1}, x_{0}, x_{1}, \cdots\right\} x_{n}$ is called the $n$th coordinate of $\omega$ and shall be considered as a function on $\Omega$ to $X$. Let $T$ be the shift transformation on $\Omega$ to $\Omega$ : the $n$th coordinate of $T \omega$ is equal to the $n+1$ th coordinate of $\omega$. For any function $g$ on $\Omega, T g$ is the function defined by $T g(\omega)=g(T \omega)$ so that $T x_{n}=x_{n+1}$. We shall consider two probability measures $\mu, \nu$ defined on $\mathscr{F}_{0}$ Let $\Omega_{n}=\prod_{i=1}^{n} X_{i}$ where $X_{i}=X, \quad i=1,2, \cdots, n$ and $\mathscr{F}_{n}=\prod_{i=1}^{n} \mathscr{S}_{i}$ where $\mathscr{S}_{i}=\mathscr{S}_{,} \quad i=$

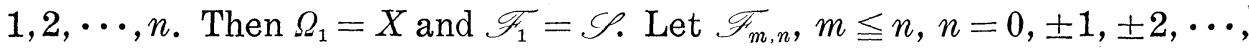
be the $\sigma$-algebra of subsets of $\Omega$ consisting of sets of the form

$$
\left[\omega=\left\{\cdots, x_{-1}, x_{0}, x_{1}, \cdots\right\}:\left(x_{m}, x_{m+1}, \cdots, x_{n}\right) \in E\right]
$$

where $E \in \mathscr{F}_{n-m+1}$. Let $\mathscr{F}_{-\infty, n}$ be the $\sigma$-algebra generated by $\bigcup_{m=-1}^{-\infty} \mathscr{F}_{m, n}$. Let $\mu_{m, n}, \nu_{m, n}$ be the contractions of $\mu, \nu$, respectively, to $\mathscr{F}_{m, n}$ and $\mu_{-\infty, n}$, $\nu_{-\infty, n}$ be the contractions of $\mu, \nu$, respectively, to $\mathscr{F}_{-\infty, n}$. Throughout this paper $\nu_{m, n}$ is assumed to be absolutely continuous with respect to $\mu_{m, n}, \nu_{m, n} \ll \mu_{m, n}$, for $m<n, n=0, \pm 1, \pm 2, \cdots$. Let $f_{m, n}$ be the derivative of $\nu_{m, n}$ with respect to $\mu_{m, n}, f_{m, n}=d \nu_{m, n} / d \mu_{m, n} . f_{m, n}$ is $\mathscr{F}_{m, n}$ measurable and nonnegative. $f_{m, n}$ is also positive with $\nu$ probability one. Hence $1 / f_{m, n}$ is well defined with $\nu$ probability one. A fundamental theorem of Information Theory by Shannon and McMillan may be considered as a theorem concerning the asymptotic properties of $f_{m, n}$ as $n \rightarrow \infty$. The theorem may be stated as follows: Let $X$ be a finite set of $K$ points and $\mathcal{S}$ be the $\sigma$-algebra of all subsets of $X$. Let $\nu$ be any stationary ( $T$ invariant) probability measure on $\mathscr{F}$ and $\mu$ be the equally distributed independent (product) measure. Then $n^{-1} \log f_{1, n}$ converges in $L_{1}(\nu)$. In particular, if $\nu$ is ergodic, the limit function is equal to $\log K-H$ with $\nu$ probability one where $H$ is the entropy of $\nu$ measure [3] [8]. Generalizations to arbitrary $X, \mathscr{S}$ were first studied by A. Pérez. He introduced an $A_{\mu}$ condition on $\nu$ as follows. $\nu$ is said to satisfy $A_{\mu}$ condition if $\nu_{-\infty, n}$ is absolutely continuous with respect to $\nu_{-\infty, 0}, \mu_{1, n}$ for $n=1,2, \cdots$. He proved the following theorem. If $\nu, \mu$ are stationary and $\mu$ is the product (independent) measure on $\mathscr{F}$ and if

Received May 31, 1960. This work was supported in part by a summer research grant from Wayne State University. 
(a) $\lim _{n \rightarrow \infty} n^{-1} \int \log f_{1, n} d \nu$ exists and is finite,

(b) $\nu$ satisfies condition $A_{\mu}$, then $\left\{n^{-1} \log f_{1, n}\right\}$ converges in $L_{1}(\nu)$ [6]. Later Pérez announced that the theorem remains to be true for any stationary measures $\mu, \nu$ [8]. The present writer proved that for Markovian $\mu, \nu$ with $\nu$ being stationary and $\mu$ having stationary transition probabilities the $\nu$-integrability of $\log f_{1,2}$ implies the $L_{1}(\nu)$ convergence of $\left\{n^{-1} \log f_{1, n}\right\}$. The proof is based on an iteration formula for $f_{1, n}$ [4]. In this paper we shall study the case that $\nu$ is stationary and $\mu$ is Markovian with stationary transition probabilities. It shall be proved that the condition

(c) $\int\left(\log f_{1, n}-\log f_{1, n-1}\right) d \nu \leqq M<\infty$ for $n=1,2,3, \ldots$ implies the $L_{1}(\nu)$ convergence of $\left\{n^{-1} \log f_{1, n}\right\}$. In fact the conditions (c) and (a) are equivalent for this case, so that the theorem is a generalization of the theorem of Pérez given in [6]. The proof is conducted along similar lines used by McMillan. The crucial step is proving the $L_{1}(\nu)$ convergence of $\left\{\log f_{-n, 0}-\log f_{-n,-1}\right\}$. The condition (c) is shown to be necessary and sufficient for this convegence.

2. Generalizations of Shannon-McMillan theorem. Let $x, \mathscr{S}, \Omega$, $\mathscr{F}, \Omega_{n}, \mathscr{F}_{n}, \mathscr{F}_{m, n}, \mu_{m, n}, \nu_{m, n}, f_{m, n}$ be as in $I$. Notations for conditional probabilities and conditional expectations relative to one or several random variables will be as in [2], Chapter $1, \S 7$. A probability measure on $\mathscr{F}$ is Markovian if, for any $A \in \mathscr{S}, m<n n=0, \pm 1, \pm 2, \cdots$

$$
P\left[x_{n} \in A \mid x_{m}, \cdots, x_{n-1}\right]=P\left[x_{n} \in A \mid x_{n-1}\right]
$$

with probability one. A Markovian measure is said to have stationary transition probabilities if for any $A \in \mathscr{S}$ and any integer $n$

$$
P\left[x_{n} \in A \mid x_{n-1}\right]=T^{n} P\left[x_{0} \in A \mid x_{-1}\right]
$$

with probability one. In this paper, since we have two probability measures $\mu, \nu$, we need to use subscripts $\mu, \nu$ to indicate conditional probabilities and conditional expectations taken under $\mu, \nu$ respectively. For any $E \subset \Omega, I_{E}$, the indicator of $E$, is the real valued function on $\Omega$ defined by

$$
\begin{array}{rlll}
I_{E}(\omega)=1 & \text { if } & & \omega \in E \\
=0 & \text { if } & & \omega \notin E .
\end{array}
$$

The log in this paper is the logarithm with base 2 .

Lemma 1. Define $\nu_{m, n}^{\prime}$ on $\mathscr{F}_{m, n}$ by

$$
\nu_{m, n}^{\prime}(E)=\int P_{\mu}\left[E \mid x_{m}, \cdots, x_{n-1}\right] d \nu,
$$


then $\nu_{m, n}^{\prime}$ is a probability measure on $\mathscr{F}_{m, n}$ with $\nu_{m, n}^{\prime}(E)=\nu_{m, n}(E)$ for $E \in \mathscr{F}_{m, n-1}$. Furthermore $\nu_{m, n} \ll \nu_{m, n}^{\prime}$ with

$$
d \nu_{m, n}\left|d \nu_{m, n}^{\prime}=f_{m, n}\right| f_{m, n-1} .
$$

Proof.

$$
\begin{aligned}
\nu_{m, n}^{\prime}(E) & =\int P_{\mu}\left[E \mid x_{m}, \cdots, x_{n-1}\right] d \nu \\
& =\int P_{\mu}\left[E \mid x_{m}, \cdots, x_{n-1}\right] f_{m, n-1} d \mu \\
& =\int E_{\mu}\left[I_{E} f_{m, n-1} \mid x_{m}, \cdots, x_{n-1}\right] d \mu \\
& =\int_{E} f_{m, n-1} d \mu .
\end{aligned}
$$

Hence $\nu_{m, n}^{\prime}$ is a probability measure on $\mathscr{F}_{m, n}$. Furthermore, for $E \in \mathscr{F}_{m, n}$

$$
\begin{aligned}
\nu_{m, n}(E) & =\int_{E} f_{m, n} d \mu=\int_{E}\left(f_{m, n} \mid f_{m, n-1}\right) f_{m, n-1} d \mu \\
& =\int_{E}\left(f_{m, n} \mid f_{m, n-1}\right) d \nu_{m, n}^{\prime} .
\end{aligned}
$$

Hence $\nu_{m, n}$ is absolutely continuous with respect to $\nu_{m, n}^{\prime}$ and $d \nu_{m, n} / d \nu_{m, n}^{\prime}=$ $f_{m, n} \mid f_{m, n-1}$.

THEOREM 1. If $\nu$ is stationary and $\mu$ is Markovian with stationary transition probabilities then

$$
f_{m, n} \mid f_{m, n-1}=T^{n}\left(f_{m-n, 0} \mid f_{m-n,-1}\right)
$$

with $\nu$ probability one for all $m<n, n=0, \pm 1, \pm 2, \cdots$.

Proof. If $\mu$ is Markovian and has stationary transition probabilities then for any $A \in \mathscr{S}$,

$$
\begin{aligned}
P_{\mu}\left[x_{n} \in A \mid x_{m}, \cdots, x_{n-1}\right] & =P_{\mu}\left[x_{n} \in A \mid x_{n-1}\right] \\
& =T^{n} P_{\mu}\left[x_{0} \in A \mid x_{-1}\right]
\end{aligned}
$$

with $\mu$ probability one and, therefore, also with $\nu$ probability one. Hence for any $A \in \mathscr{S}, B \in \mathscr{F}_{n-m}$

$$
\begin{aligned}
\nu_{m, n}^{\prime}\left[x_{n}\right. & \left.\in A,\left(x_{m}, \cdots, x_{n-1}\right) \in B\right] \\
& =\int_{\left[\left(x_{m}, \cdots, x_{n-1}\right) \in B\right]} P_{\mu}\left[x_{n} \in A \mid x_{m}, \cdots, x_{n-1}\right] d \nu \\
& =\int_{\left[\left(x_{m}, \cdots, x_{n-1}\right) \in B\right]} P_{\mu}\left[x_{n} \in A \mid x_{n-1}\right] d \nu
\end{aligned}
$$




$$
\begin{aligned}
& =\int_{\left[\left(x_{m}, \cdots, x_{n-1}\right) \in B\right]} T^{n} P_{\mu}\left[x_{0} \in A \mid x_{-1}\right] d \nu \\
& =\int_{\left[\left(x_{m-1}, \cdots, x_{-1}\right) \in B\right]} P_{\mu}\left[x_{0} \in A \mid x_{-1}\right] d \nu \\
& =\int_{\left[\left(x_{m-n}, \cdots, x_{-1}\right) \in B\right]} P_{\mu}\left[x_{0} \in A \mid x_{m-n}, \cdots, x_{-1}\right] d \nu \\
& =\nu_{m-n, 0}^{\prime}\left[x_{0} \in A,\left(x_{m-n}, \cdots, x_{-1}\right) \in B\right] .
\end{aligned}
$$

It follows that

$$
\nu_{m, n}^{\prime}\left[\left(x_{m}, \cdots, x_{n}\right) \in C\right]_{a}^{\prime \prime}=\nu_{m-n, 0}^{\prime}\left[\left(x_{m-n}, \cdots, x_{0}\right) \in C\right]
$$

for every $C \in \mathscr{F}_{n-m+1}$. Since by Lemma 1

$$
d \nu_{m, n}\left|d \nu_{m, n}^{\prime}=f_{m, n}\right| f_{m, n-1}, d \nu_{m-n, 0} / d \nu_{m-n, 0}^{\prime}=f_{m-n, 0} / f_{m-n,-1}
$$

(2) follows easily.

LEMMA 2. If $\mu$ is Markovain and $m_{1}<m_{2}<0$ then $\nu_{m_{1}, 0}^{\prime}$ is an extension of $\nu_{m_{2}, 0}^{\prime}$ to $\mathscr{F}_{m_{1}, 0}$.

Proof. For any $A \in \mathscr{S}, \beta \in \mathscr{F}_{-m_{2}}$

$$
\begin{aligned}
\nu_{m_{1}, 0}^{\prime}\left[x_{0}\right. & \left.\in A,\left(x_{m_{2}}, \cdots, x_{-1}\right) \in B\right] \\
& =\int_{\left[\left(x_{m_{2}}, \cdots, x_{-1}\right) \in B\right]} P_{\mu}\left[x_{0} \in A \mid x_{m_{1}}, \cdots, x_{-1}\right] d \nu \\
& =\int_{\left[\left(x_{m_{2}}, \cdots, x_{-1}\right) \in B\right]} P_{\mu}\left[x_{0} \in A \mid x_{-1}\right] d \nu \\
& =\int_{\left[\left(x_{m_{2}}, \cdots, x_{-1}\right) \in B\right]} P_{\mu}\left[x_{0} \in A \mid x_{m_{2}}, \cdots, x_{-1}\right] d \nu \\
& =\nu_{m_{2}, 0}^{\prime}\left[x_{0} \in A,\left(x_{m_{2}}, \cdots, x_{-1}\right) \in B\right] .
\end{aligned}
$$

It follows that

$$
\nu_{m_{1}, 0}(E)=\nu_{m_{2}, 0}(E)
$$

for every $E \in \mathscr{F}_{m_{2}, 0}$.

THEOREM 2. If $\mu$ is Markovian and $m_{1}<m_{2}<0$ then

$$
\begin{aligned}
\int\left(\log f_{m_{1}, 0}\right. & \left.-\log f_{m_{1},-1}\right) d \nu \\
& \geqq \int\left(\log f_{m_{2}, 0}-\log f_{m_{2},-1}\right) d \nu \geqq 0 .
\end{aligned}
$$

Proof. By Lemma $2 \nu_{m_{1}, 0}^{\prime}$ is an extension of $\nu_{m_{2}, 0}^{\prime}$ to $\mathscr{F}_{m_{1}, 0}$. Since $\nu_{m_{1}, 0} \ll \nu_{m_{1}, 0}^{\prime}, \nu_{m_{2}, 0} \ll \nu_{m_{2}, 0}^{\prime}$ by Lemma $1, d \nu_{m_{2}, 0} / d \nu_{m_{2}, 0}^{\prime}$ is the conditional expectation of $d \nu_{m_{1}, 0} / d \nu_{m_{1}, 0}^{\prime}$ relative to $\mathscr{F}_{m_{2}, 0}$ under the measure $\nu_{m_{1} .0}^{\prime}$. Jensen's 
inequality for conditional expectation implies that

$$
\begin{aligned}
0 & \leqq \int\left(d \nu_{m_{2}, 0} / d \nu_{m_{2}, 0}^{\prime}\right) \log \left(d \nu_{m_{2}, 0} / d \nu_{m_{2}, 0}^{\prime}\right) d \nu_{m_{1}, 0}^{\prime} \\
& \leqq \int\left(d \nu_{m_{1}, 0} / d \nu_{m_{1}, 0}^{\prime}\right) \log \left(d \nu_{m_{1}, 0} / d \nu_{m_{1}, 0}^{\prime}\right) d \nu_{m_{1}, 0}^{\prime} .
\end{aligned}
$$

Hence

$$
0 \leqq \int \log \left(d \nu_{m_{2}, 0} / d \nu_{m_{2}, 0}^{\prime}\right) d \nu \leqq \int \log \left(d \nu_{m_{1}, 0} / d \nu_{m_{1}, 0}\right) d \nu
$$

and (3) follows from (4) and Lemma 1.

THEOREm 3. If $\mu$ is Markovian then $\left\{\log f_{m, 0}-\log f_{m,-1}\right\}$ converges with $\nu$ probability one as $m \rightarrow-\infty$. The limit function may take $\pm \infty$ as its values.

Proof. It is sufficient to prove that $\left\{f_{m,-1} / f_{m, 0}\right\}$ converges with $\nu$ probability one as $m \rightarrow-\infty$. Since $\nu_{m, 0}$ is absolutely continuous with respect to $\nu_{m, 0}^{\prime}$ and $d \nu_{m, 0} / d \nu_{m, 0}^{\prime}=f_{m, 0} / f_{m,-1}$ by Lemma $1, f_{m,-1} / f_{m, 0}$ is the derivative of $\nu_{m, 0}$ continuous part of $\nu_{m, 0}^{\prime}$ with respect to $\nu_{m, 0}$. Since, by Lemma 2, $\nu_{m_{1}, 0}^{\prime}$ is an extension of $\nu_{m_{1}, 0}^{\prime}$ if $m_{1}<m_{2},\left\{-f_{-k,-1} \mid f_{-k, 0}, \mathscr{F}_{-k, 0}\right.$, $k \geqq 1\}$ is a $\nu$ semimartingale ([2] pp. 632). Since

$$
\int\left|-f_{-k,-1}\right| f_{-k, 0}\left|d \nu=\int f_{-k,-1}\right| f_{-k, 0} d \nu \leqq 1
$$

the semimartingale convergence theorem implies that $\left\{f_{-k-1} \mid f_{-k, 0}\right\}$ converges with $\nu$ probability one as $k \rightarrow \infty$.

The following lemma may be considered as an improvement of a theorem by A. Pérez ([6] Theorem 7; pp. 194).

Lemma 3. Let $\beta_{1} \subset \beta_{2} \subset \cdots$ be a sequence of $\sigma$-algebras of subsets of $\Omega$ and $\beta$ be the $\sigma$-algebra generated by $\bigcup_{k} \beta_{k}$. Let $\phi, \lambda$ be two probability measures defined on $\beta$ and $\phi_{k}, \lambda_{k}$ be the contractions of $\phi, \lambda$, respectively, to $\beta_{k}$. If $\phi_{k}$ is absolutely continuous with respect to $\lambda_{k}$ for $k=1,2, \cdots$ and if there is a finite number $M$ such that

$$
\int \log \left(d \phi_{k} / d \lambda_{k}\right) d \phi \leqq M
$$

for $k=1,2, \cdots$ then

(i) $\phi$ is absolutely continuous with respect to $\lambda$,

(ii) $\log (d \phi / d \lambda)$ is $\phi$ integrable and there exists

$$
\lim _{k \rightarrow \infty} \int \log \left(d \phi_{k} / d \lambda_{k}\right) d \phi=\int \log (d \phi / d \lambda) d \phi,
$$


(iii) $\left\{\log \left(d \phi_{k} / d \lambda_{k}\right)\right\}$ converges in $L_{1}(\phi)$ to $\log (d \phi / d \lambda)$.

Proof.

(i) Let $h_{k}=d \phi_{k} / d \lambda_{k}$. Then $\left\{h_{k}, \beta_{k}, k \geqq 1\right\}$ is a martingale under $\lambda$ measure. Now

$$
M \geqq \int \log \left(d \phi_{k} / d \lambda_{k}\right) d \phi=\int\left(\log h_{k}\right) h_{k} d \lambda .
$$

and

$$
M+\frac{1}{2} \geqq \int\left(h_{k} \log h_{k}+\frac{1}{2}\right) d \lambda \geqq(\log n) \int_{\left(h_{n} \leqq n\right)} h_{k} d \lambda .^{1}
$$

Hence

$$
\int_{\left(h_{k} \leqq n\right)} h_{k} d \lambda \leqq(\log n)^{-1}\left(M+\frac{1}{2}\right)
$$

so that $\int_{\left(h_{k} \geqq n\right)} h_{k} d \lambda \rightarrow 0$ as $n \rightarrow \infty$, uniformly in $k$. Hence $\left\{h_{k}\right\}$ converges with $\lambda$ probability one and also in $L_{1}(\lambda)$ ([2] Theorem 4.1, pp. 319). Let the limit function be $h$. Then $\int_{A} h d \lambda=\phi(A)$ for all $A \in \bigcup_{k} \beta_{k}$ and so for all $A \in \beta$. This proves that $\phi$ is absolutely continuous and that $h=(d \phi \mid d \lambda)$.

(ii) The sequence $\left\{h_{k} \log h_{k}\right\}$ converges with $\lambda$ probability one to $h \log h$. Since the functions $h_{k} \log h_{k}$ are bounded below uniformly by the number $\frac{1}{2}$,

$$
\int h \log h d \lambda \leqq \underline{\lim } \int h_{k} \log h_{k} d \lambda=\underline{\lim } \int \log h_{k} d \phi \leqq M .
$$

Hence $h \log h$ is $\lambda$ integrable. Since the real valued function $\xi \log \xi$ is continuous and convex, $h_{1} \log h_{1}, h_{2} \log h_{2}, \cdots, h \log h$ constitute a semimartingale under the measure $\lambda([2]$, Theorem 1.1, pp. 295). Hence

$$
\int h_{1} \log h_{1} d \lambda \leqq \int h_{2} \log h_{2} d \lambda \leqq \cdots \leqq h \log h d \lambda ，
$$

so that $\lim _{k \rightarrow \infty} \int h_{k} \log h_{k} d \lambda$ exists and is equal to $\int h \log h d \lambda$. Now

$$
\int|\log h| d \phi=\int h|\log h| d \lambda=\int|h \log h| d \lambda,
$$

hence $\log h$ is $\phi$ integrable and

(6) $\int \log h d \phi=\int h \log h d \lambda=\lim _{k \rightarrow \infty} \int h_{k} \log h_{k} d \lambda=\lim _{k \rightarrow \infty} \int \log h_{k} d \phi$.

${ }^{1}$ Inequality (5) was pointed out by the referee. The proof of Lemma 3 was much shortened by following his suggestions. 
(iii) Since $h_{1} \log h_{1}, h_{2} \log h_{2}, \cdots, h \log h$ constitute a semimartingale under the measure $\lambda$, we have, for $E \in \beta_{k}$,

$$
\int_{E} h_{k} \log h_{k} d \lambda \leqq \int_{E} h_{k+1} \log h_{k+1} d \lambda \leqq \int_{E} h \log h d \lambda .
$$

Hence

$$
\int_{E} \log h_{k} d \phi \leqq \int_{E} \log h_{k+1} d \phi \leqq \int_{E} \log h d \phi,
$$

so that $\log h_{1}, \log h_{2}, \cdots, \log h$ constitute a semimartingale under the measure $\phi$. Hence (ii) implies that $\log h_{k}$ are uniformly $\phi$ integrable and $\left\{\log h_{k}\right\}$ converges to $\log h$ in $L_{1}(\phi)$ ([2], Theorem 4.1s, pp. 324).

THEOREM 4. If $\mu$ is Markvian and there is a finite number $M$ such that

$$
\int\left[\log f_{m, 0}-\log f_{m,-1}\right] d \nu \leqq M
$$

for $m=-1,-2, \cdots$ then $\left\{\log f_{m, 0}-\log f_{m,-1}\right\}$ converges in $L_{1}(\nu)$ as $m \rightarrow-\infty$. and

Proof. By Lemma $2 \nu_{m_{1}, 0}^{\prime}$ is an extension of $\nu_{m_{2}, 0}^{\prime}$ if $m_{1}<m_{2}<0$

$$
d \nu_{m, 0} / d \nu_{m, 0}^{\prime}=f_{m, 0} / f_{m,-1} .
$$

If there is a probability measure $\nu^{\prime}$ defined on the $\sigma$-algebra generated by $\bigcup_{m=-1}^{-\infty} \mathscr{F}_{m, 0}$ which is an extension of $\nu_{m, 0}^{\prime}$ for $m=-1,-2, \cdots$, then the conclusion of the theorem follows easily from Lemma 3 . If $X$ is the real line and if $\mathscr{S}$ is the $\sigma$-algebra of Borel sets then the existence of $\nu^{\prime}$ follows from the Consistency Theorem of Kolmogorov. For the general case we shall proceed by using the usual representation by space $\Omega^{\prime}$ of sequences of real numbers as follows:

Let

$$
g_{k}=f_{-k, 0} \mid f_{-k,-1} .
$$

Let $G$ be the map of $\Omega$ into the space $\Omega^{\prime}$ of real sequences $\left\{\xi_{1}, \xi_{2}, \cdots\right\}$ defined by

$$
G(\omega)=\left\{g_{1}(\omega), g_{2}(\omega), \cdots\right\} .
$$

Considering $\xi_{k}$ as functions on $\Omega^{\prime}$ we have

$$
\xi_{k}(G(\omega))=g_{k}(\omega) \text {. }
$$

Let $\beta_{k}$ be the collection of Borel subsets of $\Omega^{\prime}$ which are determined by conditions on $\xi_{1}, \xi_{2}, \cdots, \xi_{k}$ and $\beta$ be the collection of all Borel subsets 
of $\Omega^{\prime}$. Let $\phi$ be the probability measure on $\beta$ and $\phi_{k}, \lambda_{k}$ be the probability measures on $\beta_{k}$ defined by

$$
\begin{aligned}
\phi(E) & =\nu\left(G^{-1} E\right), \\
\phi_{k}(E) & =\nu_{-k, 0}\left(G^{-1} E\right), \\
\lambda_{k}(E) & =\nu_{-k, 0}^{\prime}\left(G^{-1} E\right) .
\end{aligned}
$$

$\left\{g_{k}\right\}$ converges in $L_{1}(\nu)$ if and only if $\left\{\xi_{k}\right\}$ converges in $L_{1}(\phi)$. Now $\lambda_{k}$ are consistent; Kolmogorov's Consistency Theorem implies the existence of a probability measure $\lambda$ on $\beta$ which is an extension of every $\lambda_{k}$ and $d \phi_{k} / d \lambda_{k}=\xi_{k}$. Hence Lemma 3 is applicable and the $L_{1}(\phi)$ convergence of $\left\{\xi_{k}\right\}$ is obtained.

Theorem 5. If $\nu$ is stationary and $\mu$ is Markovian with stationary transition probabilities and if

$$
\int \log f_{0,0} d \nu<\infty
$$

and if there is a finite number $M$ such that

$$
\int\left(\log f_{0, n}-\log f_{0, n-1}\right) d \nu \leqq M
$$

for $n=1,2, \cdots$ then $n^{-1} \log f_{0 . n}$ converges in $L_{1}(\nu)$ as $n \rightarrow \infty$. In particular, if $\nu$ is ergodic, the limit is equal to a nonnegative constant with $\nu$ probability one.

Proof. By Theorem $4\left\{\log f_{m, 0}-\log f_{m,-1}\right\}$ converges in $L_{1}(\nu)$ as $m \rightarrow-\infty$. Let $h$ be the $L_{1}(\nu)$ limit of the sequence. Let $\bar{h}$ be the $L_{1}(\nu)$ limit of the sequence $\left\{n^{-1} \sum_{i=1}^{n} T^{i} h\right\}$. By Theorem $1 f_{0, n} / f_{0, n-1}=$ $T^{n}\left(f_{-n, 0} / f_{-n,-1}\right)$, hence

$$
\begin{aligned}
& n^{-1} \log f_{0, n}=n^{-1} \log f_{0,0}+n^{-1} \sum_{i=1}^{n} T^{i} \log \left(f_{-i, 0} \mid f_{-i,-1}\right) \\
& \int\left|n^{-1} \sum_{i=1}^{n} T^{i} \log \left(f_{-i, 0} \mid f_{-i,-1}\right)-\bar{h}\right| d \nu \\
& \leqq n^{-1} \sum_{i=1}^{n} \int\left|T^{i} \log \left(f_{-i, 0} \mid f_{-i,-1}\right)-T^{i} h\right| d \nu \\
&+\int\left|n^{-1} \sum T^{i} h-\bar{h}\right| d \nu \\
&= n^{-1} \sum_{i=1}^{n} \int\left|\log \left(f_{-i, 0} \mid f_{-i,-1}\right)-h\right| d \nu \\
&+\int\left|n^{-1} \sum T^{i} h-\bar{h}\right| d \nu \rightarrow 0 \text { as } n \rightarrow \infty .
\end{aligned}
$$

Thus the $L_{1}(\nu)$ convergence of $\left\{n^{-1} \log f_{0, n}\right\}$ is proved. The limit is $\bar{h}$ 
which is the $L_{1}(\nu)$ limit of $\left\{n^{-1} \sum_{i=1}^{n} T^{i} h\right\}$. If $\nu$ is ergodic

$$
\bar{h}=\int h d \nu
$$

with $\nu$ probability one and

$$
\int h d \nu=\lim _{m \rightarrow-\infty} \int\left[\log f_{m, 0}-\log f_{m,-1}\right] d \nu \geqq 0 .
$$

CoRollary 1. Under the hypothesis of Theorem 5 if $\nu$ is stationary and ergodic but not Markovian then $\nu$ is singular to $\mu$.

Proof. If $\mu$ is Markovian but $\nu$ is not Markovian then there is a positive integer $n_{0}$ such that

$$
\mu\left[f_{0, n_{0}-1} \neq f_{0, n_{0}}\right]>0 .
$$

For, if for every positive integer $n$

$$
\mu\left[f_{0, n-1} \neq f_{0, n}\right]=0
$$

then

$$
P_{\nu}\left[x_{n} \in A \mid x_{0}, \cdots, x_{n-1}\right]=P_{\mu}\left[x_{n} \in A \mid x_{n-1}\right]
$$

with $\nu$ probability one for every $A \in \mathscr{S}$ and $\nu$ is Markovian instead. Now since

$$
f_{0, n_{0}-1}=E_{\mu}\left[f_{0, n_{0}} \mid x_{0}, \cdots, x_{n_{0}-1}\right]
$$

and the function $\xi \log \xi$ is strictly convex, hence

$$
\int f_{0 . n_{0}} \log f_{0, n_{0}} d \mu-\int f_{0, n_{0}-1} \log f_{0, n_{0}-1} d \mu>0
$$

so that

$$
\int\left[\log f_{0, n_{0}}-\log f_{0, n_{0}-1}\right] d \nu>0 \text {. }
$$

Since $\int\left[\log f_{0, n}-\log f_{0, n-1}\right] d \nu$ is non-decreasing in $n$,

$$
\lim _{n \rightarrow \infty} \int\left[\log f_{0, n}-\log f_{0, n-1}\right] d \nu=a>0 .
$$

Now $\nu$ is ergodic; the $L_{1}(\nu)$ limit $\bar{h}$ of $\left\{n^{-1} \log f_{0, n}\right\}$ is equal to $a$ with $\nu$ probability one. Let $n_{1}, n_{2}, \cdots$ be a sequence of positive integers for which $\left\{n_{k}^{-1} \log f_{0, n_{k}}\right\}$ converges with $\nu$ probability one to $a$ so that $\left\{1 / f_{0, n_{k}}\right\}$ converges to 0 as $n_{k} \rightarrow \infty$. Let $\mathscr{F}^{\prime}$ be the $\sigma$-algebra generated by $\mathrm{U}_{n} \mathscr{F}_{0, n}$ and let $\mu_{\mathscr{F}^{\prime}}, \mu_{\mathscr{F}^{\prime}}$ be the contractions of $\mu, \nu$, respectively, to $\mathscr{F}^{\prime}$. Since $1 / f_{0, n}$ is the derivative of $\nu$-continuous part of $\mu_{0, n}$ with respect 
to $\nu_{0, n},\left\{1 / f_{0, n}\right\}$ converges with $\nu$ probability one to the derivative of $\nu$-continuous part of $\mu^{\prime}$ with respect to $\nu^{\prime}$ by a theorem of Anderson and Jessen [1]. Now we have

$$
\lim _{n \rightarrow \infty} 1 / f_{1, n}=0
$$

with $\nu$ probability one and $\mu^{\prime}$ is singular to $\nu^{\prime}$. Hence $\mu, \nu$ are singular to each other.

Extensions of Theorem 5 and Corollary 1 to $K$-Markovian $\mu$ are immediate.

3. Discussion. As was mentioned in the introduction the crucial step in establishing Theorem 5 is to prove the $L_{1}(\nu)$ convergence of $\left\{\log f_{-n, 0}-\log f_{-n,-1}\right\}$. If $\mu$ is the product (independent) measure on $\mathscr{F}$ the measure $\nu^{\prime}$ in the proof of Theorem 4 is actually $\nu_{-\infty-1} \times \mu_{0,0}$. Thus condition (c) or, equivalently, condition (a) implies condition (b) in the introduction. In [7] it is stated that the condition (b) is necessary for the $L_{1}(\nu)$ convergence of $\left\{\log f_{-n, 0}-\log f_{-n,-1}\right\}$ ([7] Theorem $2(\mathrm{~b})$ ). A simple is as follows. Let $X$ be the real line and $\mathscr{S}$ be the collection of all Borel sets. Let $\nu=\mu$ and distribution of $x_{0}$ be Gaussian. Let $\nu\left(x_{0}=x_{1}\right)=\mu\left(x_{0}=x_{1}\right)=1$. Then $\nu_{-1,0}$ is singular to $\nu_{-1,-1} \times \nu_{0,0}$, however the $L_{1}(\nu)$ convergence of $\left\{\log f_{-n, 0}-\log f_{-n-1}\right\}$ is trivially true since $f_{m, n} \equiv 1$.

\section{REFERENCES}

1. Erik Sparre Anderson and Borge Jessen, Some limit theorems in an abstract set, Danske Vic. Selsk. Nat.-ys. Medd. 22, No. 14 (1946).

2. J. L. Doob, Stochastic Processes, John Wiley and Sons, Inc., New York.

3. B. McMillan, The basic theorems of information theory, Annals of Math. Statistics, 24 (1953), 196-219.

4. Shu-Teh C. Moy, Asymptotic properties of derivatives of stationary measures, Pacific J. Math., 10 (1960), 1371-1383.

5. A. Pérez, Sur la théorie de l'information dans le cas d'un alphabet abstrait, Transactions of the First Prague Conference on information theory, statistical decision functions, random processes, Publishing House of the Czechoslavak Academy of Sciences, Prague, (1957) 183208.

6. - Sur la convergence des incertitudes, entropies et informations échantillon (sample) vers leurs vraies, Transactions of the First Prague Conference on information theory, statistical decisions functions, random processes, Publishing House of Czechoslavak Academy of Sciences, Prague (1957), 209-243.

7 - Information theory with an abstract alphabet, generalized forms of McMillan's limit theorem for the case of discrete and continuous times, Theory of Probability and its Applications, (An English translation of the Soviet Journal Teoriya Veryoatnostei i ee Primeneniya) Vol. 4, No. 1 (1959), 99-102.

8. C. E. Shannon, The mathematical theory of communication, Bell System Technical Journal, 27 (1948), 379-423, 623-656. 


\title{
PACIFIC JOURNAL OF MATHEMATICS
}

\author{
EDITORS
}

\author{
Ralph S. Phillips \\ Stanford University \\ Stanford, California \\ F. H. Brownell \\ University of Washington \\ Seattle 5, Washington
}

\author{
A. L. Whiteman \\ University of Southern California \\ Los Angeles 7, California \\ L. J. PAige \\ University of California \\ Los Angeles 24, California
}

ASSOCIATE EDITORS
E. F. BECKENBACH
D. DERRY
H. L. ROYDEN
E. G. STRAUS
T. M. CHERRY
M. OHTSUKA
E. SPANIER
F. WOLF

\section{SUPPORTING INSTITUTIONS}

\author{
UNIVERSITY OF BRITISH COLUMBIA \\ CALIFORNIA INSTITUTE OF TECHNOLOGY \\ UNIVERSITY OF CALIFORNIA \\ MONTANA STATE UNIVERSITY \\ UNIVERSITY OF NEVADA \\ NEW MEXICO STATE UNIVERSITY \\ OREGON STATE COLLEGE \\ UNIVERSITY OF OREGON \\ OSAKA UNIVERSITY \\ UNIVERSITY OF SOUTHERN CALIFORNIA
}

\author{
STANFORD UNIVERSITY \\ UNIVERSITY OF TOKYO \\ UNIVERSITY OF UTAH \\ WASHINGTON STATE COLLEGE \\ UNIVERSITY OF WASHINGTON \\ * * * * \\ AMERICAN MATHEMATICAL SOCIETY \\ CALIFORNIA RESEARCH CORPORATION \\ HUGHES AIRCRAFT COMPANY \\ SPACE TECHNOLOGY LABORATORIES \\ NAVAL ORDNANCE TEST STATION
}

Mathematical papers intended for publication in the Pacific Journal of Mathematics should be typewritten (double spaced), and the author should keep a complete copy. Manuscripts may be sent to any one of the four editors. All other communications to the editors should be addressed to the managing editor, L. J. Paige at the University of California, Los Angeles 24, California.

50 reprints per author of each article are furnished free of charge; additional copies may be obtained at cost in multiples of 50 .

The Pacific Journal of Mathematics is published quarterly, in March, June, September, and December. The price per volume (4 numbers) is $\$ 12.00$; single issues, $\$ 3.50$. Back numbers are available. Special price to individual faculty members of supporting institutions and to individual members of the American Mathematical Society: $\$ 4.00$ per volume; single issues, $\$ 1.25$.

Subscriptions, orders for back numbers, and changes of address should be sent to Pacific Journal of Mathematics, 103 Highland Boulevard, Berkeley 8, California.

Printed at Kokusai Bunken Insatsusha (International Academic Printing Co., Ltd.), No. 6, 2-chome, Fujimi-cho, Chiyoda-ku, Tokyo, Japan.

\section{PUBLISHED BY PACIFIC JOURNAL OF MATHEMATICS, A NON-PROFIT CORPORATION}

The Supporting Institutions listed above contribute to the cost of publication of this Journal, but they are not owners or publishers and have no responsibility for its content or policies. 


\section{Pacific Journal of Mathematics}

\section{Vol. 11, No. 2 December, 1961}

Tsuyoshi Andô, Convergent sequences of finitely additive measures........

Richard Arens, The analytic-functional calculus in commutative topological algebras..........................................

Michel L. Balinski, On the graph structure of convex polyhedra in

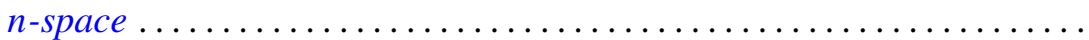

R. H. Bing, Tame Cantor sets in $E^{3}$...

Cecil Edmund Burgess, Collections and sequences of continua in the plane.

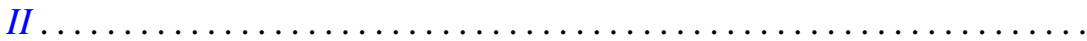

J. H. Case, Another 1-dimensional homogeneous continuum which contains an $\operatorname{arc}$

Lester Eli Dubins, On plane curves with curvature ................. 471

A. M. Duguid, Feasible flows and possible connections .............. 483

Lincoln Kearney Durst, Exceptional real Lucas sequences ................ 489

Gertrude I. Heller, On certain non-linear opeartors and partial differential equations........................................

Calvin Virgil Holmes, Automorphisms of monomial groups

Wu-Chung Hsiang and Wu-Yi Hsiang, Those abelian groups characterized by their completely decomposable subgroups of finite rank ..........

Bert Hubbard, Bounds for eigenvalues of the free and fixed membrane by finite difference methods .........................

D. H. Hyers, Transformations with bounded mth differences. .

Richard Eugene Isaac, Some generalizations of Doeblin's decomposition

John Rolfe Isbell, Uniform neighborhood retracts ..........

Jack Carl Kiefer, On large deviations of the empiric D. F. of vector chance variables and a law of the iterated logarithm...

Marvin Isadore Knopp, Construction of a class of modular functions and forms. II. . .

Gunter Lumer and R. S. Phillips, Dissipative operators in a Banach space....

Nathaniel F. G. Martin, Lebesgue density as a set function ...

Shu-Teh Chen Moy, Generalizations of Shannon-McMillan theorem ...

Lucien W. Neustadt, The moment problem and weak convergence in $L^{2}$

Kenneth Allen Ross, The structure of certain measure algebras...

James F. Smith and P. P. Saworotnow, On some classes of scalar-product algebras.

Dale E. Varberg, On equivalence of Gaussian measures. . 